\section{The makings of a marathon}

\section{H. C. Bennet-Clark}

Microscopic Anatomy of Invertebrates, Vols 1-3 of 15. Edited by F. W. Harrison and John O. Corliss/Jane A. Westfall/ Burton J. Bogitsch. Wiley-Liss/Wiley: 1990/1991. Pp. 493/436/347. Each $\$ 150, £ 116.50$.

THE idea of reviewing the first few volumes of what is intended eventually to be a 15 -volume series on invertebrate anatomy is one that would fill most with gloom. Uppermost is the fear of having to wade through endless irrelevant or unintelligible photomicrographs. The difference in this case, though, is that an important and valuable work of scholarship has been achieved.

A work of this type relies on the quality of the material presented, which in turn depends on the strength of the team of authors and the uniformity of overall editorial control. So what is the overall impression? The presentation is attractive, the quality of paper is high, and the reproduction, whether of line drawings or photomicrographs, is clean and detailed: publication expense has not been stinted, and this shows in the mostly excellent illustrations, many of them reproduced here for the first time. The pictures complement a series of useful descriptive texts, each supplemented by extensive bibliographies and taxonomic and subject indices.

What has gone wrong? Happily, fairly little, but I found the layout of the volume on Protozoa confusing and, from chapter to chapter, inconsistent. A contents page for each chapter would have helped me to find my way around. For example, the chapter on Mastigophora starts with a treatment of size, shape and symmetry, whereas that on Ciliophora starts with ecology and moves on to evolution and taxonomy. This would have been fine had I easily been able to locate the taxonomy section for Mastigophora or the microanatomy section for Ciliophora.

And another quibble: although most of the illustrations have either a scale or details of magnification, the microscopic technique used is not always stated. Light macrophotographs sometimes jostle promiscuously with transmission and scanning electron micrographs - yes, of course we can usually work out which is which, but for consistency we should be told.

Series of chapters treat the different groups: Protista are covered in four chapters and Cnidaria (polyps, corals, sea-anemones, jellyfish, hydra) in three, but Porifera (sponges) and Ctenophora (comb-jellies, sea acorns) have chapters to themselves. This division of the taxa into broad systematic units leads to a comparative anatomical survey within each taxon.

The first volume tackles Protozoa, but not in an entirely contemporary way. Indeed, do we still recognize the Protozoa in 1991? In an introductory chapter, J. O. Corliss discusses the problems of splitting or clumping the various taxa of eukaryotic microorganisms. $\mathrm{He}$ emerges with a working compromise between the extremes of splitting into more than 20 unlinked phyla and the nowarchaic clumping into one phylum with difficulty with a work such as this, although in this respect the chapter on Hydrozoa is far better.

So on to the third volume: Platyhelminthes (flat worms) and Nemertinea (ribbon worms). Of the Platyhelminthes, free-living turbellarians receive as much treatment as parasitic flukes and tapeworms together, despite the greater importance of the last two classes as the cause of diseases such as schistosomiasis and fascioliasis, as well as hydatid cysts. But much of this apparent imbalance results from having to deal with the problems of the taxonomic diversity of the Turbellaria, leading to a rather confusing text that is light on illustrations.

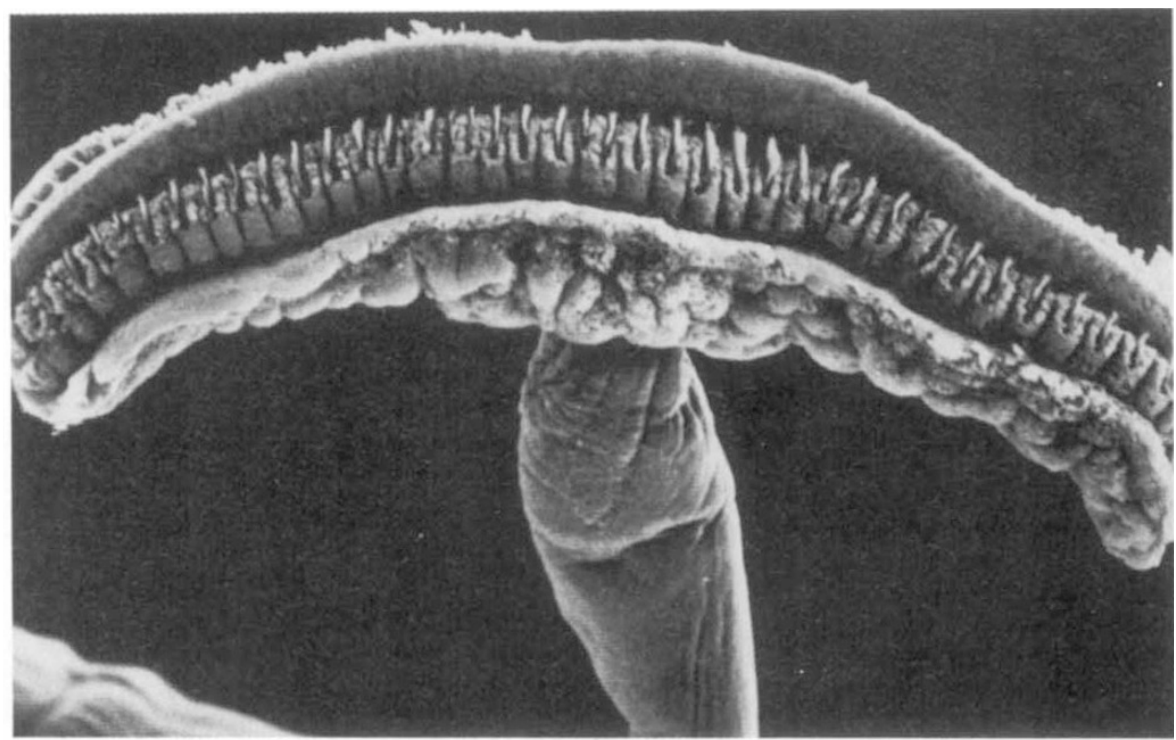

Gripping stuff - Scanning electron micrograph of the clamp of Cathetocephalus thatcheri, a tapeworm that infects the sandbar shark. Magnification, $\times 4,650$.

the 'protozoan' classes of flagellates, amoebae, sporozoans and ciliates. These clumpings are used to divide the volume into chapters, but the 'five kingdoms' phyla are used to divide within the chapters, so one must have a working knowledge of the taxonomy of protists to unravel the differences that appear between the anatomy of the Prymnesiophyta and the Euglenophyta. Fortunately Corliss provides a guide, but it is still not easy to browse.

Far more accessible is the second volume, covering Placozoa, Porifera, Cnidaria and Ctenophora. I particularly commend the exciting treatment of the Hydrozoa that ranges far beyond microanatomy into aspects of morphogenesis, functional morphology and physiology. The volume opens with a brief but useful account of the four phyla and thereafter the chapter-by-chapter treatment is logical and consistent. Although the micrographs and descriptions are excellent, the chapter on Ctenophora is let down by a lack of general or threedimensional diagrams or small-scale micrographs next to the highly magnified details of anatomy: this is a common
The chapter would have been improved greatly by a lengthier and more descriptive systematic introduction. In the chapter on flukes, there is amazingly no decent illustration of a ciliated first-stage larva (miracidium) of Digenea (liver flukes), even though the anatomy of the final larva (cercaria) and adult are well and diversely illustrated. The high point of the volume has to be the chapter on tapeworms: excellent scanning electron micrographs of attachment mechanisms and of the body surface; admirable diagrams, description and photomicrographs of the body wall; and intimate details of the reproductive system.

What a marathon. The editor is to be congratulated for keeping a fairly tight editorial hand on the enterprise but more for having the idea in the first place: he has succeeded in getting world leaders in each field as his authors and they, in turn, have pulled together an immense and valuable amount of information.

H. C. Bennet-Clark is in the Department of Zoology, University of Oxford, South Parks Road, Oxford OX1 3PS, UK 